\title{
Thermodynamic instabilities and strangeness production in hot and dense hadronic matter
}

\section{A. Lavagno*}

Department of Applied Science and Technology, Politecnico di Torino, and INFN Torino, Italy E-mail: andrea.lavagno@polito.it

\begin{abstract}
One of the very interesting aspects in the high energy heavy-ion collisions experiments and in nuclear astrophysics is a detailed study of the thermodynamic properties of strongly interacting nuclear matter far away from the nuclear ground state. The main goal of this contribution is to show that thermodynamic instabilities and phase transitions can take place at finite net baryon density and temperature, where the onset conditions of deconfined quark-gluon plasma should not still realized. Similarly to the low density nuclear liquid-gas phase transition, we show that a finite density phase transition is characterized by pure hadronic matter with both mechanical instability (fluctuations on the baryon density) that by chemical-diffusive instability (fluctuations on the strangeness concentration). The main goal is to investigate how the constraints on the global conservation of the baryon number, electric charge fraction, and strangeness neutrality, in the presence of Delta-isobar degrees of freedom, hyperons, and strange mesons, influence the behavior of the equation of state in a regime of finite values of baryon density and temperature. It turns out that in this situation hadronic phases with different values of strangeness content may coexist, altering significantly meson-antimeson ratios.
\end{abstract}

The European Physical Society Conference on High Energy Physics

5-12 July, 2017

Venice

${ }^{*}$ Speaker. 
The relativistic mean-field model is widely successful used for describing the properties of finite nuclei as well as hot and dense nuclear matter [1, 2, 3, 4]. The Lagrangian for the selfinteracting octet of baryons can be written as [1]

$$
\begin{aligned}
\mathscr{L}_{\text {octet }}= & \sum_{k} \bar{\psi}_{k}\left[i \gamma_{\mu} \partial^{\mu}-\left(M_{k}-g_{\sigma k} \sigma\right)-g_{\omega k} \gamma_{\mu} \omega^{\mu}-g_{\rho k} \gamma_{\mu} \vec{t} \cdot \vec{\rho}^{\mu}\right] \psi_{k} \\
& +\frac{1}{2}\left(\partial_{\mu} \sigma \partial^{\mu} \sigma-m_{\sigma}^{2} \sigma^{2}\right)-\frac{1}{3} a\left(g_{\sigma N} \sigma\right)^{3}-\frac{1}{4} b\left(g_{\sigma N} \sigma^{4}\right) \\
& +\frac{1}{2} m_{\omega}^{2} \omega_{\mu} \omega^{\mu}+\frac{1}{4} c\left(g_{\omega N}^{2} \omega_{\mu} \omega^{\mu}\right)^{2}+\frac{1}{2} m_{\rho}^{2} \vec{\rho}_{\mu} \cdot \vec{\rho}^{\mu} \\
& -\frac{1}{4} F_{\mu \nu} F^{\mu \nu}-\frac{1}{4} \vec{G}_{\mu \nu} \vec{G}^{\mu \nu}+\bar{\psi}_{\Delta v}\left[i \gamma_{\mu} \partial^{\mu}-\left(M_{\Delta}-g_{\sigma \Delta} \sigma\right)-g_{\omega \Delta} \gamma_{\mu} \omega^{\mu}\right] \psi_{\Delta}^{\nu},
\end{aligned}
$$

where the sum runs over the full octet of baryons and $\psi_{\Delta}^{v}$ is the Rarita-Schwinger spinor for the $\Delta$-isobars $\left(\Delta^{++}, \Delta^{+}, \Delta^{0}, \Delta^{-}\right)[5,6,7]$. The adopted coupling constants are the same of Ref. [8].

We are dealing with the study of a multi-particle system at finite temperature and density with two conserved charges: baryon (B) number and zero net strangeness ( $S$ ) number $\left(r_{S}=\rho_{S} / \rho_{B}=0\right.$ ). For what concern the electric charge $(\mathrm{Q})$, we work in symmetric nuclear matter with a fixed value of $Z / A=0.5$ and we do not consider fluctuations in the electric charge fraction, due to the high temperature regime considered in the present investigation [9]. Therefore, the electric charge results to be separately conserved in each phase during the phase transition and the chemical potential of particle of index $i$ can be written as $\mu_{i}=b_{i} \mu_{B}+s_{i} \mu_{S}$, where $b_{i}$ and $s_{i}$ are, respectively, the baryon and the strangeness quantum numbers of $i$-th hadronic species.

Assuming the presence of two phases (denoted as $I$ and $I I$, respectively), the system is stable against the separation in two phases if the free energy of a single phase is lower than the free energy in all two phases configuration. The phase coexistence is given by the Gibbs conditions

$$
\begin{aligned}
& \mu_{B}^{I}=\mu_{B}^{I I}, \quad \mu_{S}^{I}=\mu_{S}^{I I}, \\
& P^{I}\left(T, \mu_{B}, \mu_{S}\right)=P^{I I}\left(T, \mu_{B}, \mu_{S}\right) .
\end{aligned}
$$

At a given baryon density $\rho_{B}$ and at a given zero net strangeness density $r_{S}=\rho_{S} / \rho_{B}=0$, the chemical potentials $\mu_{B}$ are $\mu_{S}$ are univocally determined by the following equations

$$
\begin{aligned}
& \rho_{B}=(1-\chi) \rho_{B}^{I}\left(T, \mu_{B}, \mu_{S}\right)+\chi \rho_{B}^{I I}\left(T, \mu_{B}, \mu_{S}\right), \\
& \rho_{S}=(1-\chi) \rho_{S}^{I}\left(T, \mu_{B}, \mu_{S}\right)+\chi \rho_{S}^{I I}\left(T, \mu_{B}, \mu_{S}\right),
\end{aligned}
$$

where $\rho_{B}^{I(I I)}$ and $\rho_{S}^{I(I I)}$ are, respectively, the baryon and strangeness densities in the low density (I) and in the higher density (II) phase and $\chi$ is the volume fraction of the phase $I I$ in the mixed phase $(0 \leq \chi \leq 1)$ [10]. An important feature of this conditions is that, unlike the case of a single conserved charge, baryon and strangeness densities can be different in the two phases, although the total $\rho_{B}$ and $\rho_{S}$ are fixed.

For such a system in thermal equilibrium, the possible phase transition can be characterized by mechanical (fluctuations in the baryon density) and chemical instabilities (fluctuations in the strangeness number). As usual the condition of the mechanical stability implies

$$
\rho_{B}\left(\frac{\partial P}{\partial \rho_{B}}\right)_{T, \rho_{S}}>0 .
$$


By introducing the notation $\mu_{i, j}=\left(\partial \mu_{i} / \partial \rho_{j}\right)_{T, P}$ (with $i, j=B, S$ ), the chemical stability can be expressed with the following conditions [8]

$$
\mu_{B, B}>0, \quad \mu_{S, S}>0 .
$$

In addition to the above conditions, for a process at constant $P$ and $T$, it is always satisfied that

$$
\begin{aligned}
& \rho_{B} \mu_{B, B}+\rho_{S} \mu_{S, B}=0, \\
& \rho_{B} \mu_{B, S}+\rho_{S} \mu_{S, S}=0 .
\end{aligned}
$$

Whenever the above stability conditions are not respected, the system becomes unstable and the phase transition take place. The coexistence line of a system with one conserved charge becomes in this case a two dimensional surface in $\left(T, P, r_{S}\right)$ space, enclosing the region where mechanical and diffusive instabilities occur [8].

In Fig. 1, we show the pressure as a function of baryon density at fixed temperature. The continuous (dashed) lines correspond to the solution obtained with (without) the Gibbs construction. In this case the conditions of mechanical and chemical stability are not satisfied and the system goes to a phase transition. Let us observe that, although the system has globally zero net strangeness $\left(r_{S}=0\right)$, the mixed phase is realized with two phase with different and finite values of strangeness and different baryon-antibaryon content (baryon and strangeness charges are globally conserved but are different in the two hadronic phases).

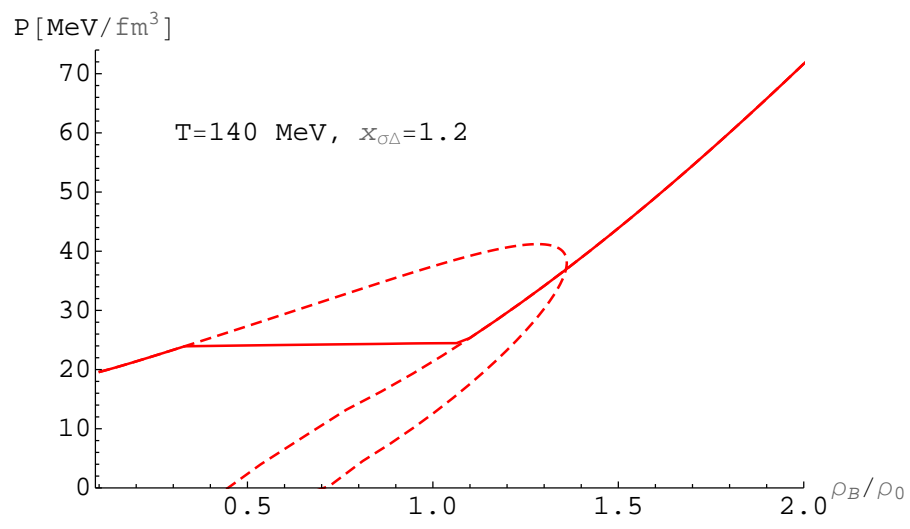

Figure 1: Pressure at fixed temperature as a function of baryon density ( $\rho_{0}$ is the nuclear saturation density). The continuous (dashed) lines correspond to the solution obtained with (without) the Gibbs construction.

The above feature imply remarkably consequences in the antiparticle to antiparticle production and in the strangeness content at different temperatures and baryon densities. In Fig. 2, right panel, the antiproton to proton ratios $R$, as a function of the baryon density, are reported for different temperatures $\left(x_{\sigma \Delta}=g_{\sigma \Delta} / g_{\sigma N}=1.2\right.$ is the used $\sigma$ meson- $\Delta$ ratio coupling constant and $x_{\omega \Delta}=$ $\left.g_{\omega \Delta} / g_{\omega N}=1\right)$. The black points in the isothermal curves stand for the beginning and the end of the phase transition. We observe that at lower $(T \leq 100 \mathrm{MeV})$ and higher $(T \geq 150 \mathrm{MeV})$ temperatures, the phase transition does not occur, while, in the intermediate range of temperature $(100<T<150$ $\mathrm{MeV}$ ), thermodynamic instabilities take place and this feature can imply an abrupt variation of the ratio during the phase transition region. Consistently, in Fig. 2, left panel, we report the strangeness 
fraction $Y$ for particle and antiparticle as a function of baryon density at fixed temperature $T=140$ $\mathrm{MeV}$. During the phase transition (between the two vertical dashed lines) a strong variation in the antibaryon-barion ratio takes place with a significant increase of antibaryon $(\bar{B})$ particles. This feature is reflected in the strangeness fraction with a stronger increase in positive values of $Y_{S}$ due to antibaryon $\bar{B}$ (antihyperon) particles with respect to negative values due to baryons $B$ (hyperons). Also meson $M$ and antimeson $(\bar{M})$ particles undergo to fluctuation in the strangeness fraction but this feature appears to be less pronounced.
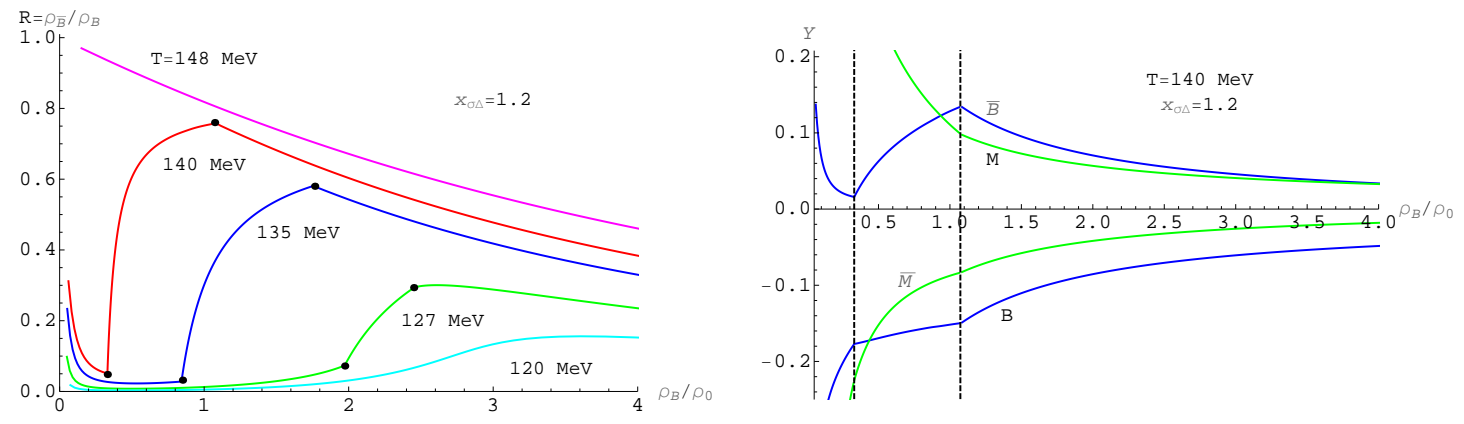

Figure 2: Left panel: Antiproton to proton ratios as a function of baryon density at different temperatures. The black points stand for the beginning and the end of the phase transition. Right panel: Strangeness fractions $Y$ of baryons (B), mesons (M) and their antiparticles as a function of baryon density in the pure hadronic phase and in the mixed phase at fixed temperature and zero net strangeness.

In conclusion, similarly to the liquid-gas phase transition in a warm and low density nuclear matter, a pure hadronic phase transition also can occur at higher temperatures and densities due to the presence of both mechanical and chemical instabilities. During the phase transition the two hadronic phases have a different baryon density and different strangeness fraction in the mixed phase, although the global strangeness is fixed to zero. This feature could be phenomenological very relevant in order to identify such phase transition in the future compressed baryonic matter experiments.

\section{References}

[1] N.K. Glendenning and S.A. Moszkowski, Phys. Rev. Lett. 67 (1991) 2414.

[2] N.K. Glendenning and J. Schaffner-Bielich, Phys. Rev. C 60 (1999) 025803.

[3] A. Lavagno, Eur. Phys. J. A 49 (2013) 102.

[4] A. Drago, A. Lavagno, B.D. Metzger, G. Pagliara, Phys. Rev. D 93 (2016) 103001.

[5] M. Hofmann et al., Phys. Rev. C 51 (1995) 2095.

[6] Z. Li, G. Mao, Y. Zhuo, and W. Greiner, Phys. Rev. C 56 (1997) 1570.

[7] A. Lavagno, Phys. Rev. C 81 (2010) 044909.

[8] A. Lavagno, D. Pigato, Phys. Rev. C 86 (2012) 024917.

[9] G. Kaniadakis et al., Physica A 261 (1998) 359.

[10] H. Müller, B.D. Serot, Phys. Rev. C 52 (1995) 2072. 